\title{
Simulation of Thermal Explosion of Catalytic Granule in Fluctuating Temperature Field
}

\author{
Igor Derevich, Daria Galdina \\ Department of Applied Mathematics, Faculty of Fundamental Sciences, \\ Moscow State Technical University by N.E. Bauman (BMSTU), Moscow, Russian Federation \\ Email: DerevichIgor@gmail.com, GalDaria@mail.ru
}

Received July 29, 2013; revised August 29, 2013; accepted September 15, 2013

Copyright (C) 2013 Igor Derevich, Daria Galdina. This is an open access article distributed under the Creative Commons Attribution License, which permits unrestricted use, distribution, and reproduction in any medium, provided the original work is properly cited.

\begin{abstract}
Method for numerical simulation of the temperature of granule with internal heat release in a medium with random temperature fluctuations is proposed. The method utilized the solution of a system of ordinary stochastic differential equations describing temperature fluctuations of the surrounding and granule. Autocorrelation function of temperature fluctuations has a finite decay time. The suggested method is verified by the comparison with exact analytical results. Random temperature behavior of granule with internal heat release qualitatively differs from the results obtained in the deterministic approach. Mean first passage time of granules temperature intersecting critical temperature is estimated at different regime parameters.
\end{abstract}

Keywords: Stochastic Ordinary Differential Equation; Autocorrelation Function; Heat Explosion; Semenov’s Diagram; Temperature Fluctuations

\section{Introduction}

The catalytic synthesis processes are generally accompanied by heat release. Synthesis of heavy hydrocarbons in the Fischer-Tropsch process (GTL technology) is associated with essential heat generation [1]. GTL technology can solve a number of environmental and economic problems.

In the Technological Institute for Superhard and Novel Carbon Materials (Troitsk, Russian Federation), industrial reactor is developed with a capacity of $5000 \mathrm{Nm}^{3} / \mathrm{h}$ of synthesis gas with a production of $500 \mathrm{~kg} / \mathrm{h}$ stabilized liquid hydrocarbons. The reactor used fixed bed of catalyst granules.

Exothermic heat of reaction is transferred from the volume of catalytic granules to the boundary of the granules. At the boundary heat is removed to the liquid products of the synthesis. Exceeding heat generation over heat transfer leads to uncontrolled growth temperature (thermal explosion). Loss of thermal stability of catalyst granules is responsible of thermal explosion of the reactor. Therefore, investigation of critical conditions of thermal explosion is an important practical problem.

Reasons leading to thermal explosion in deterministic situation have been well studied [2-6]. There is a critical temperature, the excess of which causes a significant increase in temperature of granules. The situation drasti- cally changes when the temperature of the environment is a random process. In this case there is always a non-zero probability for a temperature fluctuation, the magnitude of which exceeds a critical value, which may lead to the loss of thermal stability. Study of the effect of random noise is dedicated to the behavior of systems with explosive features, for example, [7-11]. The results of this study can also be applicable in modeling of ignition conditions of dispersed fuel in aircraft and rocket engines, and power stations. Main trends obtained in the paper are helpful for the estimation of the probability of thermal explosion in storages and transportation lines of dispersed combustible materials.

Investigation on effect of noise is devoted to the behavior of systems with explosive behavior [7-11]. Study of random temperature fluctuations was carried out in the framework of probability density function approach [12]. This approach requires the use of modern methods of stochastic processes and functional analysis and yields results which have practical importance. However, the method of the probability density function does not take into account some important details of the complicated chemical kinetics. In this situation, it is appropriate to use the methods of modeling of temperature dynamics which is based on direct numerical solutions of stochastic ordi- 
nary differential equations [13-17].

In this paper we propose a method for direct numerical modeling of a random temperature of granule with internal heat generation with accounted temperature fluctuations in the surrounding. We construct temperature fluctuations with internal temporal structure. The autocorrelation function of temperature fluctuations of the surrounding has a finite decay time. This approach can be used in future for modeling stochastic behavior in not only temperature, but also reactant concentration inside the granule with detailed complex kinetics. Verification of the proposed algorithm is based on a comparison with exact analytical solutions. We illustrate various scenarios of the loss of thermal stability of catalytic granule. Calculations on results of the average waiting time of thermal explosion are presented.

\section{Equation for Temperature of Granule with Internal Heat Release. Semenov's Diagram}

In this section we write down the equation for the temperature of the granule with internal heat source and perform the analysis of Semenov's diagram.

\subsection{Equation for Temperature of the Catalytic Granule}

We investigate spherical granule with diameter $d_{p}$, which is placed in liquid products with temperature $\Theta_{f}$. Thermal effect of exothermal reaction inside the granule is $Q$. Rate of chemical reactions is modeled as Arrhenius law with activation energy $E$. Heat transfer coefficient is $\alpha$. Equation for the volume-averaged temperature of the granule $\Theta_{p}$ has the following form

$$
m_{p} c_{p} \frac{\mathrm{d} \Theta_{p}}{\mathrm{~d} t}=\alpha S_{p}\left(\Theta_{f}-\Theta_{p}\right)+V_{p} Q A \mathrm{e}^{-\frac{E}{R^{\circ} \Theta_{p}}} .
$$

Here $m_{p}$ is mass of the granule; $S_{p}=\pi d_{p}^{2}$ area of the granule surface; $V_{p}=\pi d_{p}^{3} / 6$ is volume of the granule; $A$ is the frequency factor; $R^{\circ}$ is the universal gas constant.

The equation for the granule temperature can be rewritten in the relaxation form

$$
\frac{\mathrm{d} \Theta_{p}}{\mathrm{~d} t}=\frac{\Theta_{f}-\Theta_{p}}{\tau_{\Theta}}+\frac{Q A}{\rho_{p} c_{p}} \mathrm{e}^{-\frac{E}{R^{\circ} \Theta_{p}}},
$$

where $\tau_{\Theta}=d_{p} c_{p} / \alpha_{p}$ is thermal relaxation time of the granule.

Temperature of the surrounding liquid is given as

$$
\Theta_{f}(t)=\left\langle\Theta_{f}\right\rangle=\theta_{f}(t),
$$

where $\left\langle\Theta_{f}\right\rangle$ is averaged temperature of the fluid; $\theta_{f}(t)$ is temperature fluctuations; $\left\langle\theta_{f}(t)\right\rangle=0$.
Angular brackets denote the results of averaging over an ensemble of random realization of fluid temperature.

Equation (1) in dimensionless variables has the form

$$
\frac{\mathrm{d} \Gamma_{p}}{\mathrm{~d} \tau}=\frac{\left(1+\gamma_{f}\right)-\Gamma_{p}}{\Omega_{\Theta}}+Q^{*} \mathrm{e}^{-\frac{E^{*}}{\Gamma_{p}}} .
$$

Here $\Gamma_{p}=\Theta_{p} /\left\langle\theta_{f}\right\rangle$ is dimensionless temperature of the granule; $\tau=t / T_{E}$ is dimensionless time; $T_{E}$ is integral time scale of fluid temperature autocorrelation function; $\gamma_{f}=\theta_{p} /\left\langle\Theta_{f}\right\rangle$ is a dimensionless temperature fluctuation of fluid surrounding; $E^{*}=E\left\langle R^{\circ}\left\langle\Theta_{f}\right\rangle\right.$ is dimensionless activation energy; $\left.Q^{*}=Q A\right)\left(\rho_{p} c_{p}\left\langle\Theta_{f}\right\rangle\right)$ is dimensionless heat of exothermal reaction;

$\Omega_{\Theta}=\tau_{\Theta} / T_{E}$ is parameter of thermal inertia of the granule.

\subsection{Semenov's Diagram}

Based on the analysis of Semenov's diagram we show the existence of critical temperature. Infinitely small excess above the critical temperature leads to uncontrolled increase of temperature of the granule (thermal explosion).

Analysis of Semenov's diagram is provided for steadystate temperature of the liquid medium. Looking for a stationary temperature of the granule from the following equation

$$
\frac{\mathrm{d} \Gamma_{p}}{\mathrm{~d} \tau}=0, \frac{\Gamma_{p}-1}{\Omega_{\Theta}}=Q^{*} \mathrm{e}^{-\frac{E^{*}}{\Gamma_{p}}} .
$$

We introduce dimensionless power of heat transfer to the liquid phase $W_{\Omega}^{*}=\left(\Gamma_{p}-1\right) / \Omega_{\Theta}$ and dimensionless power of heat release $W_{Q}^{*}=Q^{*} \exp \left(-E^{*} / \Gamma_{p}\right)$.

Figure 1 represent Semenov's diagram. It is evident that there is a region with three stationary temperatures of the granule. This region with three roots of Equation (3) is bounded by the tangential lines, whose position is determined by the values of thermal relaxation parameter of the granule.

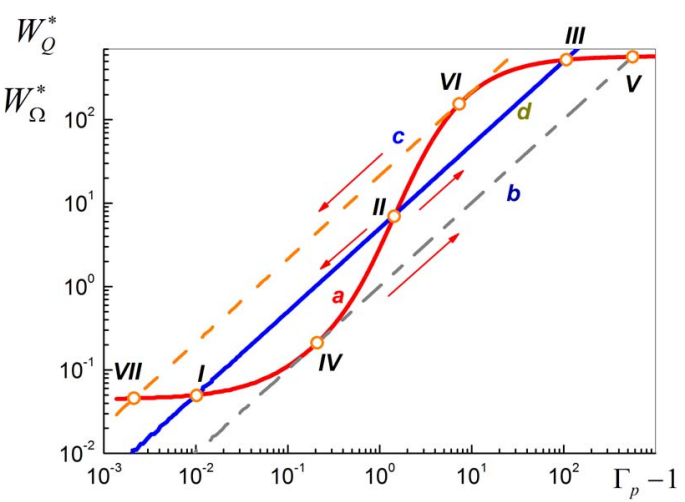

Figure 1. Semenov's diagram. 
At the tangential lines b and $c$ in Figure 1 the temperature of the granule returns to a steady state with low or high temperatures, respectively. To study the types of stationary temperature we performed numerical integration of the nonlinear Equation (2) without taking into account fluctuations in the temperature of the medium.

Figure 2 illustrates the dynamics of change of temperature of the granule, if the initial temperature is close to the second root on the Semenov's diagram. It can be seeing, that infinitely small disturbance above $\Gamma_{I I}$ give a loss of thermal stability of the granule.

If initial temperature of the granule is infinitively less than the value $\Gamma_{I I}$, the temperature of the granule proceeds to low value close to ambient temperature. The second root at the Semenov's diagram may be regarded as critical value $\Gamma_{c r}=\Gamma_{I I}$.

\section{Autocorrelation Function of Temperature Fluctuations. Exact Results}

In this section, we obtain some exact results for comparison with data of numerical simulation. Exact solutions exist for linear equations. We consider the equation for the fluctuations of temperature of the granule (1) without the chemical heat source

$$
\frac{\mathrm{d} \theta_{p}(t)}{\mathrm{d} t}=\frac{\theta_{f}(t)-\theta_{p}(t)}{\tau_{\Theta}} .
$$

Temperature fluctuations of fluid $\theta_{f}(t)$ is statisticcally stationary random process with correlation

$$
\left\langle\theta_{f}\left(t^{\prime}\right) \theta_{f}\left(t^{\prime \prime}\right)\right\rangle=\left\langle\theta_{f}^{2}\right\rangle \Psi_{f}\left(t^{\prime}-t^{\prime \prime}\right) .
$$

We use the relationship between the autocorrelation function and its spectrum

$$
\begin{aligned}
& \Psi_{f}(t)=\frac{1}{(2 \pi)} \int_{-\infty}^{\infty} \mathrm{e}^{-i \omega t} \psi_{f}(\omega) \mathrm{d} \omega, \\
& \psi_{f}(\omega)=\int_{-\infty}^{\infty} \mathrm{e}^{i \omega s} \Psi_{f}(s) \mathrm{d} s .
\end{aligned}
$$

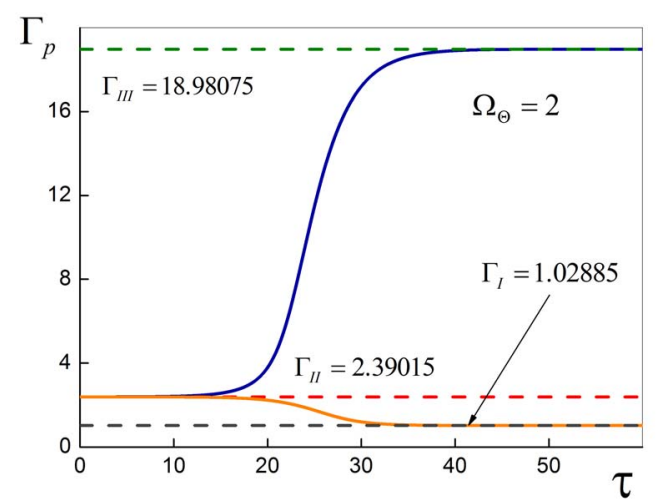

Figure 2. Temperature of the granule with initial value near second root on Semenov's diagram.
Solution of Equation (4) has the form

$$
\theta_{p}(t)=\frac{1}{\tau_{\Theta}} \int_{0}^{t} \mathrm{e}^{-\frac{t-s}{\tau_{\Theta}}} \theta_{f}(s) \mathrm{d} s .
$$

Correlation function of temperature fluctuations of the granule is written as

$$
\begin{aligned}
& \left\langle\theta_{p}\left(t^{\prime}\right) \theta_{p}\left(t^{\prime \prime}\right)\right\rangle=\left\langle\theta_{p}^{2}\right\rangle \Psi_{p}\left(t^{\prime}-t^{\prime \prime}\right) \\
& =\frac{1}{\tau_{\Theta}} \int_{0}^{t^{\prime}} \mathrm{d} s^{\prime} \mathrm{e}^{-\frac{t^{\prime}-s^{\prime}}{\tau_{\Theta}}} \frac{1}{\tau_{\Theta}} \int_{0}^{t^{\prime \prime}} \mathrm{d} s^{\prime \prime} \mathrm{e}^{-\frac{t^{\prime \prime}-s^{\prime \prime}}{\tau_{\Theta}}}\left\langle\theta_{f}\left(s^{\prime}\right) \theta_{f}\left(s^{\prime \prime}\right)\right\rangle^{.}
\end{aligned}
$$

With the help of spectrum of the fluid temperature autocorrelation function (5) and (6) we write down expression for granule autocorrelation

$$
\left\langle\theta_{p}^{2}\right\rangle \Psi_{p}(t)=\frac{\left\langle\theta_{f}^{2}\right\rangle}{2 \pi} \int_{-\infty}^{\infty} \mathrm{e}^{-i \omega t} \frac{\psi_{f}(\omega)}{1+\left(\omega \tau_{\Theta}\right)^{2}} \mathrm{~d} \omega .
$$

Square of dispersion of the granule temperature fluctuations is follows from expression (8) at $t=0$

$$
\left\langle\theta_{p}^{2}\right\rangle=\frac{\left\langle\theta_{f}^{2}\right\rangle}{2 \pi} \int_{-\infty}^{\infty} \frac{\psi_{f}(\omega)}{1+\left(\omega \tau_{\Theta}\right)^{2}} \mathrm{~d} \omega .
$$

Let us consider two special cases of the autocorrelation function of the temperature fluctuations of the fluid.

\subsection{Delta-Correlated in Time Random Process}

Temperature fluctuations $\theta_{f}(t)$ is delta-correlated in time random process. The autocorrelation function (5) has the form

$$
\Psi_{f}\left(t^{\prime}-t^{\prime \prime}\right)=2 \tau_{\circ} \delta\left(t^{\prime}-t^{\prime \prime}\right) .
$$

Here $\tau_{\circ}$ is integral time scale

$$
\int_{0}^{\infty} \Psi_{f}(s) \mathrm{d} s=2 \tau_{0} \int_{0}^{\infty} \delta(s) \mathrm{d} s=\tau_{0} .
$$

Spectrum of autocorrelation function (9) is found from expression (7)

$$
\psi_{f}(\omega)=2 \tau_{\circ} \int_{-\infty}^{\infty} \mathrm{e}^{i \omega s} \delta(s) \mathrm{d} s=2 \tau_{\circ} .
$$

Substitution expression for the spectrum into formula (8) leads to autocorrelation function of the granule temperature fluctuations

$$
\left\langle\theta_{p}^{2}\right\rangle \Psi_{p}(t)=\left\langle\theta_{f}^{2}\right\rangle \frac{\tau_{\circ}}{\tau_{\Theta}} \mathbf{e}^{-\frac{t}{\tau_{\Theta}}} .
$$

Intensity of temperature fluctuations and autocorrelation function of granule are

$$
\left\langle\theta_{p}^{2}\right\rangle=\frac{\tau_{\circ}}{\tau_{p}}\left\langle\theta_{f}^{2}\right\rangle \ll\left\langle\theta_{f}^{2}\right\rangle, \Psi_{p}(t)=\mathrm{e}^{-\frac{t}{\tau_{\Theta}}} .
$$


Delta-correlation approach is correct for granule with high thermal inertia. Autocorrelation function of temperature fluctuations of the granule has exponential form with integral temporary scale equal to the granule relaxation time.

\subsection{Exponential Approximation of Autocorrelation Function}

Second approach is exponential approximation of fluid temperature $\theta_{f}(t)$ autocorrelation function

$$
\Psi_{f}(t)=\mathrm{e}^{-\frac{t}{T_{E}}}
$$

Spectrum of the autocorrelation function (11) follows from formula (7)

$$
\psi_{f}(\omega)=\int_{-\infty}^{\infty} \mathrm{e}^{i \omega t-\frac{|t|}{T_{E}}} \mathrm{~d} t=\frac{2 T_{E}}{1+\left(\omega T_{E}\right)^{2}}
$$

Correlation of the granule temperature fluctuation is obtained from formula (11)

$$
\left\langle\theta_{p}^{2}\right\rangle \Psi_{p}(t)=\frac{\left\langle\theta_{f}^{2}\right\rangle}{2 \pi} \int_{-\infty}^{\infty} \frac{2 T_{E} \mathrm{e}^{-i \omega t}}{\left[1+\left(\omega \tau_{\Theta}\right)^{2}\right]\left[1+\left(\omega T_{E}\right)^{2}\right]} \mathrm{d} \omega .
$$

Calculation of the above integral under theory of functions with complex variables leads to the result

$$
\left\langle\theta_{p}^{2}\right\rangle \Psi_{p}(t)=\left\langle\theta_{f}^{2}\right\rangle \frac{\mathrm{e}^{-\frac{t}{T_{E}}}-\left(\tau_{\Theta} / T_{E}\right) \mathrm{e}^{-\frac{t}{\tau_{\Theta}}}}{1-\left(\tau_{\Theta} / T_{E}\right)^{2}} .
$$

Square of dispersion of the granule temperature fluctuations is follows from Equation (12) at $t=0$

$$
\left\langle\theta_{p}^{2}\right\rangle=\frac{\left\langle\theta_{f}^{2}\right\rangle}{1+\left(\tau_{\Theta} / T_{E}\right)} .
$$

Autocorrelation function of the granule temperature fluctuation also obtained from Equation (12)

$$
\Psi_{p}(t)=\frac{\mathrm{e}^{-\frac{t}{T_{E}}}-\left(\tau_{\Theta} / T_{E}\right) \mathrm{e}^{-\frac{t}{\tau_{\Theta}}}}{1-\left(\tau_{\Theta} / T_{E}\right)} .
$$

Integral time scale of the granule temperature fluctuation is

$$
T_{\Theta}=\int_{0}^{\infty} \Psi_{p}(t) \mathrm{d} t=T_{E}+\tau_{\Theta} .
$$

One can conclude about existence of two granules types. Granule with small thermal inertia with thermal relaxation time much smaller than integral time scale of fluid temperature autocorrelation function $\tau_{\Theta} \ll T_{E}$. In that case dispersion of temperature fluctuations of the granule and fluid is close $\left\langle\theta_{p}^{2}\right\rangle \approx\left\langle\theta_{f}^{2}\right\rangle$, and integral time scale of granule temperature fluctuations is $T_{\Theta} \approx T_{E}$. For granule with high thermal inertia $\tau_{\Theta} \gg T_{E}$ dispersion of granule temperature fluctuations is less then fluid $\left\langle\theta_{p}^{2}\right\rangle \approx\left(T_{E} / \tau_{\Theta}\right)\left\langle\theta_{f}^{2}\right\rangle$. Integral time scale of granule temperature fluctuations is close to temperature relaxation time $T_{\Theta} \approx \tau_{\Theta}$, and granule autocorrelation function decays as $\Psi_{p}(t) \approx \exp \left(-t / \tau_{\Theta}\right)$ (see, also Equation (10)).

Obtained exact results will be used for testing numerical algorithm of simulation of temperature of granule in a random temperature of surroundings.

\section{System of Stochastic Differential Equations}

Analytical results show that modeling autocorrelation function with finite relaxation time is possible only on the base of stochastic ordinary equations.

Write down system of differential equations for temperature fluctuations of fluid and the granule with heat release

$$
\begin{aligned}
& \frac{\mathrm{d} \gamma_{f}(\tau)}{\mathrm{d} \tau}=\eta(\tau)-\gamma_{f}(\tau), \\
& \frac{\mathrm{d} \Gamma_{p}(t)}{\mathrm{d} \tau}=\frac{\left(1+\gamma_{f}(t)\right)-\Gamma_{p}(t)}{\Omega_{\Theta}}+Q^{*} \mathrm{e}^{-\frac{E^{*}}{\Gamma_{p}(t)}} .
\end{aligned}
$$

Here $\eta(\tau)$ is seeded Gaussian random process with delta-correlated function

$$
\left\langle\eta\left(t^{\prime}\right) \eta\left(t^{\prime \prime}\right)\right\rangle=2 \tau_{\circ}\left\langle\eta^{2}\right\rangle \delta\left(t^{\prime}-t^{\prime \prime}\right) .
$$

Integration of the system of Equations (15) and (16) is carried out by explicit Euler method

$$
\begin{gathered}
\gamma_{f}^{(n+1)}=\gamma_{f}^{(n+1)}+\Delta \tau\left\{\eta^{(n)}-\gamma_{f}^{(n)}\right\}, \\
\Gamma_{p}^{(n+1)}=\Gamma_{p}^{(n)}+\Delta \tau\left\{\frac{\left(1+\gamma_{f}^{(n+1)}\right)-\Gamma_{p}^{(n)}}{\Omega_{\Theta}}+Q^{*} \mathrm{e}^{-\frac{E^{*}}{\Gamma_{p}^{(n)}}}\right\} .
\end{gathered}
$$

Here $n$ is the number of temporary steps; random increment of seeded process is modeled as

$$
\Delta \tau \eta^{(n)}=\xi^{(n)} \sqrt{2\left\langle\eta^{2}\right\rangle \tau_{\circ} \Delta \tau}
$$

where $\xi^{(n)}$ is random realization of the normalized Gaussian process (white noise) with zero mean and unit dispersion.

Figure 3 illustrates the effect of thermal inertia of the granules on temperature fluctuations without heat source. It can be conclude that increasing the thermal inertia reduces the amplitude of temperature fluctuations of the granule.

Figure 4 shows influence of thermal inertia of the granule on dispersion of temperature fluctuations. The 
increasing thermal inertia decreases the intensity of temperature fluctuations of the granule. From the Figure 4 is also evident a satisfactory agreement between the results of calculations by the exact formula (13) and numerical data obtained by averaging random realizations of temperature.

Autocorrelation function of the granule temperature fluctuations are shown in Figure 5. It can be seen that the results of numerical simulations satisfactory agree with obtained exact results. The growth of thermal inertia increases the damping region of the autocorrelation function of the granules.

\section{Simulation of Thermal Explosion. Average Waiting time of Explosion}

This section presents results showing the various scenarios of behavior of granule temperature with internal heat

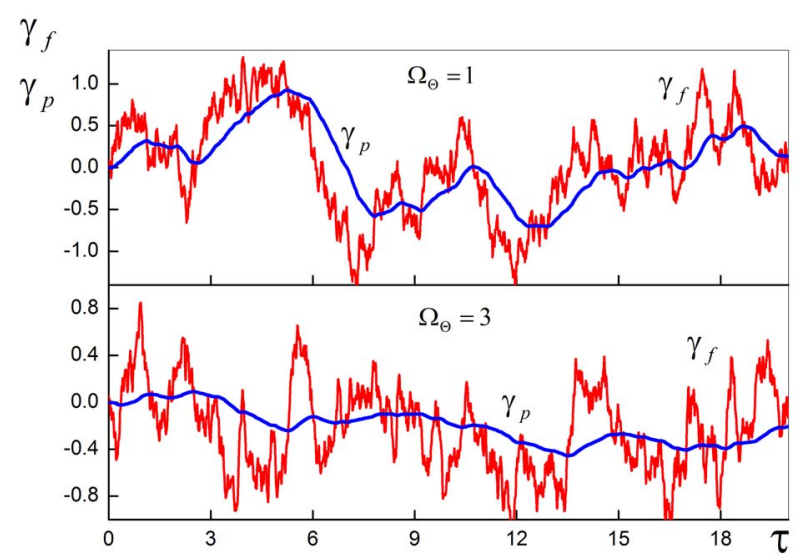

Figure 3. Random temperatures of surroundings and granule.

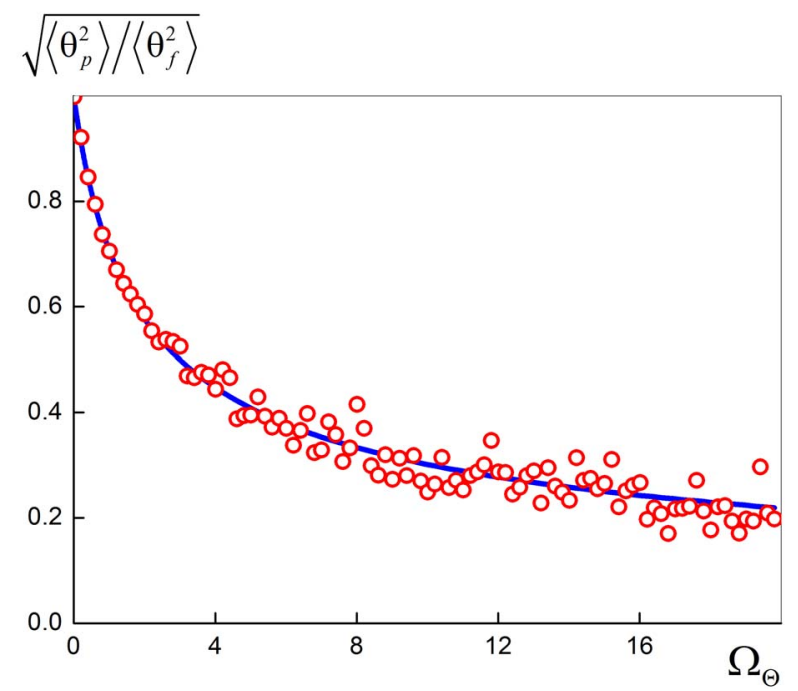

Figure 4. The ratio between dispersions of temperature fluctuations of granule and the fluid: points are simulation results; curve is the formula (13). generation with account temperature fluctuatuation of the fluid. Figure 6 shows the behavior of the actual temperature of the granules with heat generation. On the figure $\Gamma_{f}=1+\gamma_{f}(t)$ is actual temperature of surrounding fluid. It can be seen that fluctuations of magnitude of chemical reactions make a significant contribution to the value of random temperature of the granule.

On all illustrations following next the initial temperature of the granule is less than the critical value corresponding to the second root $\Gamma_{I I}$ (see Semenov's diagram).

Random process with nonzero probability may exceed any level. After some random time the actual temperature of the granule will be over the critical value $\Gamma_{c r}=\Gamma_{I I}$ and there will be a loss of thermal stability. This scenario is illustrated by Figure 7 .

The waiting time of a thermal explosion we define as the average time of first crossing by random temperature of the granule the critical level $\Gamma_{c r}$. Waiting time of

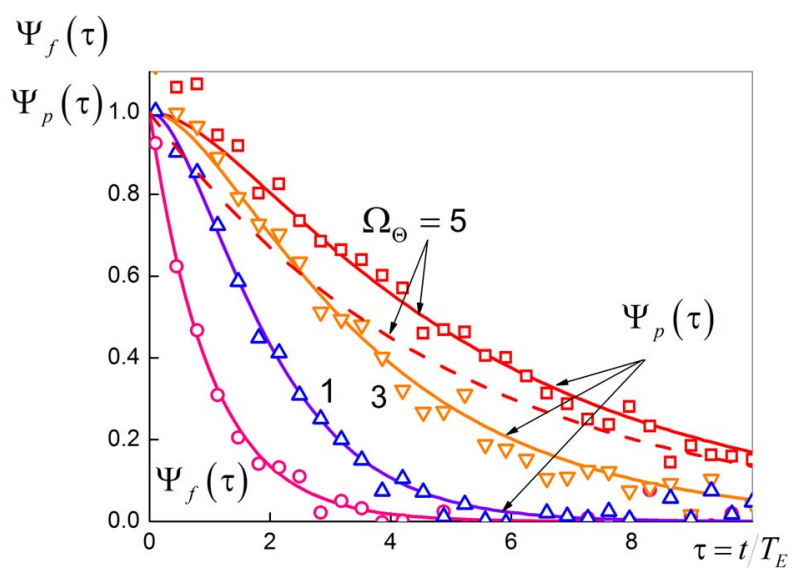

Figure 5. Autocorrelation functions of temperature fluctuations fluid and granule. Points are numerical simulations, lines are the formulas (11) and (14). Dashed line is exponential approximation (10).

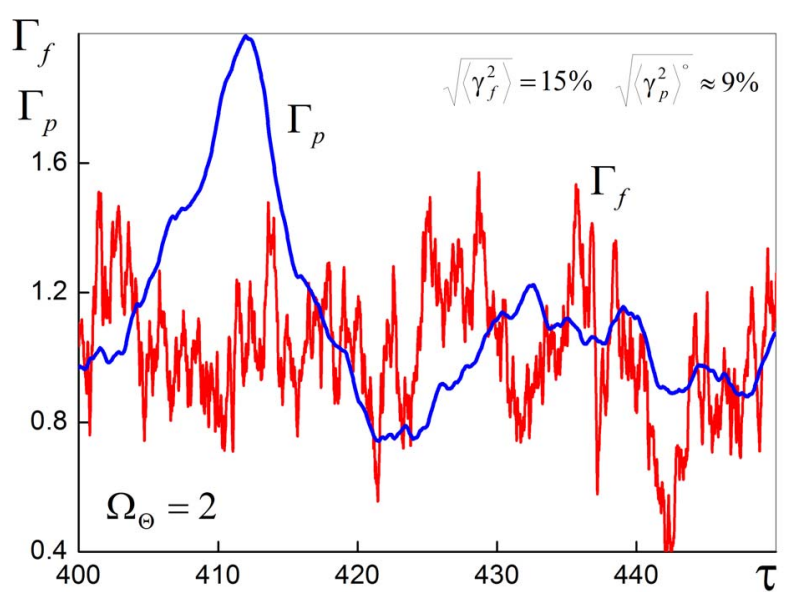

Figure 6. Example of granule temperature without heat explosion. 


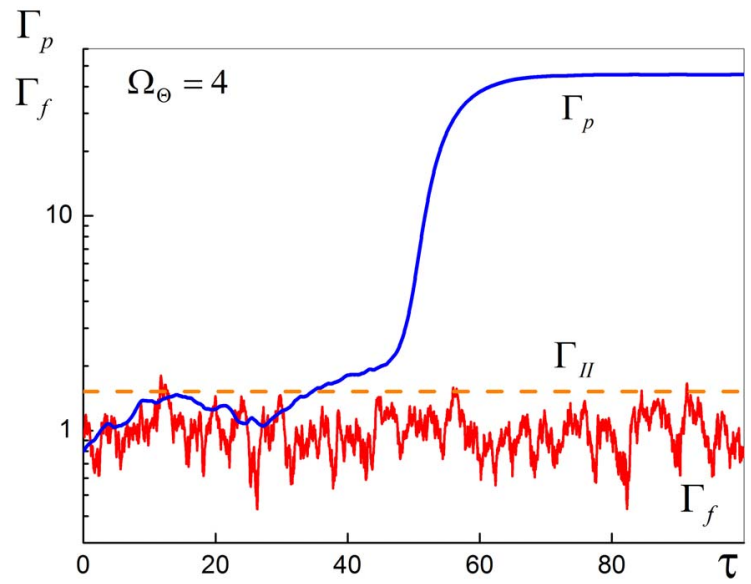

Figure 7. Example of temperature of the granule with heat explosion.

thermal explosion $\tau_{c r}$ is function of initial temperature of the granule $\Gamma_{p}^{\circ}$ (Figure 8).

As initial temperature approaches to the critical value, the average waiting time of thermal explosion dramatically reduced. The critical temperature $\Gamma_{c r}$ essentially depends on parameter of thermal inertia of the granule.

From the Figure 8 it is evident that average delay time of thermal explosion depends on the parameter of thermal relaxation of the granule.

\section{Conclusions}

Method of numerical simulation of random temperature of granules with internal heat source in surrounding liquid with temperature fluctuations is designed. The intensity of heat release is described by the Arrhenius law.

For temperature fluctuations, a numerical generation of random Gaussian process with an exponentially decaying autocorrelation function is suggested. Autocorrelation function and dispersions of temperature fluctuations without heat generation obtained by the numerical simulation are compared with the exact formulas, found by spectral analysis of stochastic processes.

Analysis of the influence of the fluid temperature fluctuations on the process of thermal explosion is carried out. Dynamics of thermal explosion for various values of granules temperature relaxation times, initial temperature of granules, and dispersion of temperature fluctuations are investigated.

Based on direct numerical simulations, the average waiting time of thermal explosion is investigated. Effect of stochastic drift of the granule temperature to its critical value is found.

Further research in the area of numerical simulation is possible to be carried out in two directions. Firstly, it is the use of the actual kinetic schemes, the Fischer-Tropsch synthesis, on cobalt catalysts. The second direction of

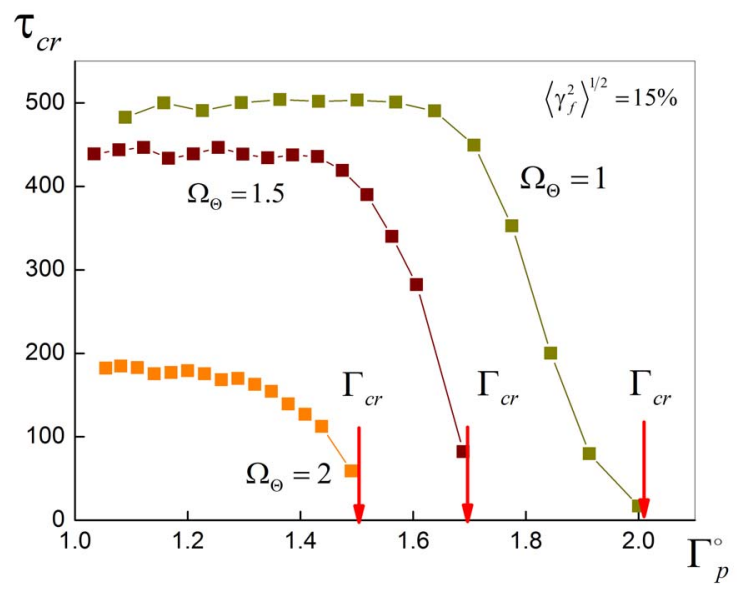

Figure 8. Average waiting time of thermal explosion.

research focuses on the accounting of the random medium temperature with intermittency, which is characterized by the log-normal distribution.

\section{Acknowledgements}

This work was supported by the Russian Foundation for Basic Research (RFBR), grant number 11-08-00645-a.

\section{REFERENCES}

[1] A. P. Steynberg, M. E. Dry, B. H. Davis and B. B. Breman, "Chapter 2-Fischer-Tropsch Reactors," Studies in Surface Science and Catalysis, Vol. 152, 2004, pp. 64195. http://dx.doi.org/10.1016/S0167-2991(04)80459-2

[2] Ya. B. Zel'dovich, G. I. Barenblatt, V. B. Librovich and G. M. Makhviladze, "Mathematical Theory of Combustion and Explosion,” Nauka, Moscow, 1980.

[3] D. A. Frank-Kamenetskii, "Diffusion and Heat Transfer in Chemical Kinetics,” Plenum, New York, 1969.

[4] A. G. Merzhanov and E. N. Rumanov, "Nonlinear Effects in Macroscopic Kinetics,” Uspekhi Fizicheskikh Nauk, Vol. 151, 1987, pp. 553-593. http://dx.doi.org/10.3367/UFNr.0151.198704a.0553

[5] J. Warnatz, U. Maas and R. W. Dibble, “Combustion. Physical and Chemical Fundamentals, Modeling and Simulations, Experiments, Pollutant Formation,” Springer, 2001.

[6] W. Horsthemke and R. Lefever, "Noise-Induced Transitions," Theory and Applications in Physics, Chemistry and Biology, Springer, 1984.

[7] I. V. Derevich and R. S. Gromadskaya, "Rate of Chemical Reactions with Regard to Temperature Fluctuations," Theoretical Foundations of Chemical Engineering, Vol. 31, No. 4, 1997, pp. 392-397.

[8] V. G. Medvedev, V. G. Telegin and G. G. Telegin, "Statistical Analysis of Kinetics of an Adiabatic Thermal Explosion," Combustion, Explosion, and Shock Waves, Vol. 45, No. 3, 2009, pp. 274-277. http://dx.doi.org/10.1007/s10573-009-0036-2 
[9] I. V. Derevich, "Temperature Oscillation in a Catalytic Particle of Fischer-Tropsch Synthesis," International Journal of Heat and Mass Transfer, Vol. 53, No. 1-3, 2010, pp. 135-153.

http://dx.doi.org/10.1016/j.ijheatmasstransfer.2009.09.045

[10] I. V. Derevich, "Effect of Temperature Fluctuations of Fluid on Thermal Stability of Particles with Exothermic Chemical Reaction," International Journal of Heat and Mass Transfer, Vol. 53, No. 25-26, 2010, pp. 5920-5932. http://dx.doi.org/10.1016/j.ijheatmasstransfer.2010.07.031

[11] I. V. Derevich, "Influence of Temperature Fluctuations on the Thermal Explosion of a Single Particle," Combustion, Explosion, and Shock Waves, Vol. 47, No. 5, 2011, pp. 538-547. http://dx.doi.org/10.1134/S0010508211050078

[12] V. I. Klyatskin, "Stochastic Equations Eyes of the Physicist: Substantive Provisions, Exact Results and Asymptotic Approaches,” FIZMATHLIT, Moscow, 2001.

[13] G. Y. Liang, L. Cao and D. J. Wu, “Approximate Fok-
ker-Planck Equation of System Driven by Multiplicative Colored Noises with Colored Cross-Correlation,” Physica A, Vol. 335, No. 3-4, 2004, pp. 371-384. http://dx.doi.org/10.1016/j.physa.2003.12.023

[14] D. T. Gillespie, "Exact Numerical Simulation of the Ornstein-Uhlenbeck Process and Its Integral,” Physical Review E, Vol. 54, No. 2, 1996, pp. 2084-2091. http://dx.doi.org/10.1103/PhysRevE.54.2084

[15] S. Ilie and A. Teslya, "An Adaptive Stepsize Method for the Chemical Langevin Equation,” Journal of Chemical Physics, Vol. 136, No. 18, 2012, Article ID: 184101.

[16] C. W. Gardiner, "Handbook of Stochastic Methods for Physics, Chemistry and the Natural Sciences,” Springer, 1983. http://dx.doi.org/10.1007/978-3-662-02377-8

[17] P. Lévy, "Processus Stochastiques et Mouvement Brownien,” Gauthier-Villars, Paris, 1965. 